DUKE-CGTP-05-05

SLAC-PUB-11261

SU-ITP-05/21

$\frac{\text { hep-th/0506041 }}{\text { June } 2005}$

\title{
Superpotentials for Quiver Gauge Theories
}

\author{
Paul S. Aspinwall ${ }^{1,2,3}$ and Lukasz M. Fidkowski ${ }^{1}$ \\ ${ }^{1}$ Department of Physics, Stanford University, Stanford, CA 94305-4060 \\ ${ }^{2}$ SLAC, Stanford, CA 94305-4060 \\ ${ }^{3}$ Center for Geometry and Theoretical Physics, Box 90318 \\ Duke University, Durham, NC 27708-0318
}

\begin{abstract}
We compute superpotentials for quiver gauge theories arising from marginal D-Brane decay on collapsed del Pezzo cycles $S$ in a Calabi-Yau $X$. This is done using the machinery of $A_{\infty}$ products in the derived category of coherent sheaves of $X$, which in turn is related to the derived category of $S$ and quiver path algebras. We confirm that the superpotential is what one might have guessed from analyzing the moduli space, i.e., it is linear in the fields corresponding to the Ext ${ }^{2} \mathrm{~s}$ of the quiver and that each such Ext ${ }^{2}$ multiplies a polynomial in Ext ${ }^{1}$ s equal to precisely the relation represented by the Ext ${ }^{2}$.
\end{abstract}




\section{Introduction}

Singularities of string backgrounds have attracted much attention and have been investigated using a variety of methods [1-6]. One is to study the gauge theory on a $D$-brane probe of the singularity. While there has been much work done on extracting gauge theory data for various types of singularities (abelian [1] and non-abelian [7] orbifolds, conifolds [8,9], toric [6,10-12] and generalized del Pezzo [13,14] singularities), a general method for determining the superpotential has been lacking. In [10] the superpotential was obtained from integrating the quiver relations for certain cases, with various ad-hoc methods being used to resolve ambiguities that arise in such an integration. In this paper, using previous work of [15], we present a general rigorous method for obtaining the superpotential of such quiver gauge theories from the quiver relations. We show that the superpotential is just the naive sum of terms of the form relation times the Ext ${ }^{2}$ field corresponding to the relation. We apply the method to the trivial example of a $\mathbb{P}^{2}$ as well as to a $\mathrm{dP}_{1}$, in which case we get a nonhomogeneous superpotential. In principle we can apply it to a general del Pezzo singularity - all we need is the del Pezzo quiver and relations.

We deal with flat compactifications $M \times X$ where $M$ is 4 dimensional Minkowski space and $X$ is a Calabi-Yau manifold, and we probe the theory with space filling branes - i.e., $(n+3)$ branes, where $n$ is the dimensionality of the brane within $X$. We expect such branes to be BPS and stable when probing a smooth point of $X$, but to marginally decay into a collection of so-called fractional branes when the point becomes singular. We consider singularities obtained when a complex surface (i.e., one that has 4 real dimensions) $S$ shrinks down to zero size in $X$ by varying the Kähler parameters. Assuming that $S$ is smooth and irreducible, it is known that $S$ must be a del Pezzo surface, i.e., $\mathbb{P}^{1} \times \mathbb{P}^{1}$, or $\mathbb{P}^{2}$ blown up at $m$ points (denoted by $\mathrm{dP}_{m}$ ), where $m$ ranges from 0 to 8 . The $3+1$ dimensional quiver gauge theory associated to this marginal decay into fractional branes is the one whose superpotential we are after. This set up has been studied extensively in the literature [9, 14, 16-23].

The moduli space of a D-brane is given by the space of critical points of the superpotential. Thus, knowing the moduli space one may make a guess at the form of the superpotential. In the case at hand, this leads to a natural conjecture for the superpotential. By using more rigorous methods we are able to show that this conjecture is correct.

For our analysis we will use the algebraic machinery of the derived category of coherent sheaves, developed in particular in $[5,24,25]$. The fractional branes have tractable representations as elements of the derived category $\mathbf{D}(X)$ of coherent sheaves on $X$. In principle the method of [15] can be applied to find the so-called $A_{\infty}$ products in the algebra of Ext groups. These $A_{\infty}$ products are determined by combinatorial relations that they have to satisfy (coming from Feynman diagrams in the associated topological theory) and they encode the superpotential. Applying the technique of [15] directly is difficult and in order to make the problem tractable, we instead proceed in two steps. First, we use a spectral sequence argument to reduce the problem from one of studying sheaves on $X$ to the simpler one of studying sheaves on $S$. It is in this reduction that we show that each Ext ${ }^{2}$ appears linearly in the superpotential, multiplying a term that involves only $\operatorname{Ext}^{1} \mathrm{~s}$ and is determined by $A_{\infty}$ relations over $S$. To compute these we exploit the well understood properties of $\mathbf{D}(S)$, and 
specifically its intimate relation with the derived category of quiver representations [26, 27]. We see that the terms involving the $\operatorname{Ext}^{1} \mathrm{~s}$ are just the (possibly non-homogeneous) relations in the quiver. It is important to note that we obtain the superpotential only up to certain nonlinear field redefinitions (see [15]) — this is the most that could be expected from such topological sigma model methods as we use.

The plan of the paper is as follows: in section 2 we review quivers and sheaves on del Pezzo surfaces, and how they relate. In section 3] we review $A_{\infty}$ algebras and the method of [15] for using the topological $B$ model to compute $A_{\infty}$ structure and hence the superpotential. In section 4 we introduce the quiver gauge theory we want to study and prove that its superpotential is linear in the $\operatorname{Ext}^{2} \mathrm{~s}$, which multiply terms determined by the $A_{\infty}$ structure over $S$ - this is the reduction from sheaves on $X$ to sheaves on $S$. In section [5 we solve the problem on $S$ by reframing it as a computation in the derived category of quiver representations, and apply the solution to the case $S=\mathbb{P}^{2}$ and the more nontrivial case $S=\mathrm{dP}_{1}$. This example illustrates the general algorithm that can be carried through for any quiver with known relations.

\section{Quivers and Sheaves}

\subsection{Quivers}

We now review the necessary mathematical notions relating to quivers and their representations. Further background can be found in [5]. First, a quiver is a directed graph $Q$ that consists of nodes $v_{i}$ and arrows $a_{\alpha}$. Its path algebra $A$ is defined as follows: as a vector space, $A$ is generated by all of the paths constructed through concatenation of arrows in $Q$. The product structure of $A$ is defined on these generators as follows: if path 1 ends on the same node that path 2 begins on, the product is defined to be the obvious concatenation; otherwise it is defined to be 0 . Note that corresponding to each node $v_{i}$ we have a corresponding zero length path and hence an idempotent element $e_{i}$ of $A$.

In the remainder of the paper, we will deal with a slight generalization to a quiver with relations. This is just a quiver whose path algebra is defined as the above $A$ quotiented out by a subspace generated by linear combinations of paths called relations. We stipulate that any given relation must be a linear combination of paths between the same two nodes. It does not, however, need to be homogeneous. A simple example (with homogeneous relations) is the so-called Beilinson quiver, defined as:

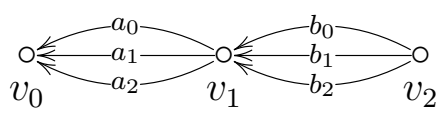

with relations $a_{\alpha} b_{\beta}-a_{\beta} b_{\alpha}$.

For a given quiver $Q$, we can consider the associated category $A-\bmod$ of left $A$-modules. For any left $A$-module $V$ we can form the vector spaces $V_{i}=e_{i} V$, of dimension $N_{i}$; if we then think of each $V_{i}$ as living on node $v_{i}$ then we see that multiplication by any arrow $a_{\alpha}$ acts as a linear transformation between the spaces $V_{i}$ at the tail and head of $a_{\alpha}$, and these 
linear transformations respect the relations. This structure is known as a representation of the quiver $Q$, of dimension $\left(N_{i}\right)$.

A map $\phi$ between left $A$-modules $V$ and $W$ is simply a linear transformation that commutes with the action of $A$, i.e., $\phi(a v)=a \phi(v)$. If we think of $V$ and $W$ as quiver representations then this condition is just the obvious constraint that the maps from $V_{i}$ to $W_{i}$ must commute with the linear transformations induced by the arrows, i.e., $\phi$ is a map of representations. Thus we sometimes refer to the category of left $A$-modules as the category of quiver representations, and we use these terms interchangeably from now on.

Corresponding to each node of $Q$ there are two distinguished representations $P_{i}$ and $L_{i}$; when we make the connection to sheaves on del Pezzo surfaces below, these will correspond to sheaves in the exceptional collection and fractional branes, respectively, as we will see. $L_{i}$ is defined simply as the one dimensional representation with $V_{i}=\mathbb{C}$ and all other $V_{j}=0$. $P_{i}$ is defined as the subspace of $A$ generated by all paths that begin at $v_{i}$; it is trivially seen to be a subrepresentation. It may seem that $P_{i}$ is a rather large representation, and indeed, if the quiver has any loops there will be infinite dimensional $P_{i}$. However, in the case of quivers associated to del Pezzo's there will be an ordering on the nodes that is respected by the arrows and hence all $P_{i}$ will be finite-dimensional. This finite dimensionality is in large part responsible for the tractability of the problem and motivates the reduction mentioned earlier from sheaves on the Calabi-Yau to sheaves on the del Pezzo. In the Beilinson quiver, for example, we see that $P_{0}, P_{1}$, and $P_{2}$ have dimensions $(1,0,0),(3,1,0)$, and $(6,3,1)$ respectively.

When we make the connection between quivers and sheaves it will be through the derived category. Before we talk about that, however, let us first discuss some basic homological properties of quivers. First of all, one can show that the $P_{i}$ are projective objects in $A-\bmod$. In fact, they form a complete set in the sense that any left $A$-module has a resolution by various direct sums of these $P_{i}$. These projective resolutions can be used to compute higher Ext groups. For example, the projective resolution of any $L_{i}$ is

$$
\cdots \longrightarrow \bigoplus_{k} P_{k}^{\oplus r_{i k}} \longrightarrow \bigoplus_{k} P_{k}^{\oplus n_{i k}} \longrightarrow P_{i} \longrightarrow L_{i} \longrightarrow 0 .
$$

Here $n_{i j}$ is the number of arrows from node $i$ to node $j$ and $r_{i j}$ is the number of independent relations imposed on paths from $i$ to $j$. In the case of the Beilinson quiver, the resolutions are:

$$
\begin{array}{r}
0 \longrightarrow P_{0} \longrightarrow L_{0} \longrightarrow 0 \\
0 \longrightarrow P_{0}^{\oplus 3} \longrightarrow P_{1} \longrightarrow L_{1} \longrightarrow 0 \\
0 \longrightarrow P_{0}^{\oplus 3} \longrightarrow P_{1}^{\oplus 3} \longrightarrow P_{2} \longrightarrow L_{2} \longrightarrow 0
\end{array}
$$

Noting that $\operatorname{Ext}^{k}$ is the $k$ th derived functor of $\operatorname{Hom}$ and that $\operatorname{Hom}\left(P_{i}, L_{j}\right)=\delta_{i j} \mathbb{C}$ we can compute $\operatorname{Ext}^{p}(A, B)$ by taking a projective resolution

$$
\ldots \longrightarrow \Pi_{2} \longrightarrow \Pi_{1} \longrightarrow \Pi_{0} \longrightarrow A \longrightarrow 0,
$$


where the $\Pi_{i}$ are direct sums of $P_{i}$ 's and from it constructing the complex

$$
0 \longrightarrow \operatorname{Hom}\left(\Pi_{0}, B\right) \longrightarrow \operatorname{Hom}\left(\Pi_{1}, B\right) \longrightarrow \operatorname{Hom}\left(\Pi_{2}, B\right) \longrightarrow \ldots
$$

The cohomology of this complex in the $p$ th position is then $\operatorname{Ext}^{p}(A, B)$. Using this method one can show that

$$
\begin{aligned}
& \operatorname{dim} \operatorname{Ext}^{1}\left(L_{i}, L_{j}\right)=n_{i j} \\
& \operatorname{dim} \operatorname{Ext}^{2}\left(L_{i}, L_{j}\right)=r_{i j} .
\end{aligned}
$$

In general, higher Ext's may also exist. For example, Ext ${ }^{3}$ represents relations amongst relations. However, for the purposes of quiver gauge theories, the appearance of higher Ext's is unphysical $[16,28]$ and so we assume

$$
\operatorname{Ext}^{k}\left(L_{i}, L_{j}\right)=0, \quad k \geq 3
$$

Finally, we note that we can recover the quiver path algebra from the projective representations $P_{i}$. Supposing $Q$ is a quiver with $n$ nodes, this is done as follows: we let

$$
T=P_{0} \oplus P_{1} \oplus \cdots \oplus P_{n-1} .
$$

Using the fact that $\operatorname{Hom}\left(P_{i}, P_{j}\right)$ is simply the vector space of paths from $j$ to $i$ we can verify that

$$
A \cong \operatorname{End}(T)^{\mathrm{op}} \text {. }
$$

In other words, $A$ is just the algebra $\operatorname{End}(T)$ with the product structure reversed.

\subsection{The Derived Category}

Having reviewed this preliminary material about quiver representations we move on to briefly discuss the derived category. As mentioned above, the derived category will form a bridge between the quiver representations that we have already discussed and the category of coherent sheaves introduced below. One source is [5]; here we just review the facts we will use.

Given any abelian category $\mathscr{A}$ (such as that of quiver representations, or that of coherent sheaves discussed below) we can define its derived category $\mathbf{D}(\mathscr{A})$ as follows. The objects in $\mathbf{D}(\mathscr{A})$ are complexes of objects in $\mathscr{A}$ :

$$
\ldots \longrightarrow \mathscr{E}^{0} \stackrel{d_{0}}{\longrightarrow} \mathscr{E}^{1} \stackrel{d_{1}}{\longrightarrow} \mathscr{E}^{2} \stackrel{d_{2}}{\longrightarrow} \ldots
$$

To construct the morphisms in $\mathbf{D}(\mathscr{A})$, we begin with the abelian group of all possible maps between complexes (not necessarily respecting the differential). These maps are graded by their degree $p$ and can be written as

$$
\sum_{n} f_{n, n+p}
$$


where $f_{m, n}$ is a map from $\mathscr{E}^{m}$ to $\mathscr{E}^{n}$. We define a differential on this group by (abusing notation slightly):

$$
(d f)_{n, p+1}=d_{n+p} f_{n, p}-(-1)^{p} f_{n+1, p} d_{n}
$$

The derived morphisms are now defined as the cohomology of this group, with formal inverses added in for all quasi-isomorphisms (that is, those chain maps which induce isomorphisms on cohomology).

Now we state some necessary results without proof. Given any object $A$ in $\mathscr{A}$, we can construct the associated one term complex whose only nonzero entry is $A$, at the zeroth position. For brevity we will henceforth refer to both the object and the associated one term complex by $A$. Then, for $A, B$ in $\mathscr{A}$, Ext $\left.\operatorname{Ex}^{\bullet} A, B\right)$ is given by the group of derived morphisms between the complexes associated to $A$ and $B$, with the grading on Ext corresponding to the grading of the derived morphisms. In fact, this is the generalization of the notion of Ext to the arbitrary elements of $\mathbf{D}(\mathscr{A})$. Also, any $A$ in $\mathscr{A}$ is equivalent to its projective or injective resolution in the derived category. Further, if we represent either $A$ by its projective resolution or $B$ by its injective resolution then the generators of $\operatorname{Ext}(A, B)$ can be written as honest chain maps between these complexes.

\section{$2.3 \quad$ Sheaves}

We now turn to reviewing key aspects of the other relevant category, that of coherent sheaves. It turns out that (as we will see in more detail below) the derived category of coherent sheaves on a Calabi-Yau manifold $X$, denoted $\mathbf{D}(X)$, precisely describes $D$-branes in the topological B model defined on $X$. The open string modes stretching between them are described by the Ext groups of the sheaf homs between the relevant branes [5, 24,25]. These, in turn, describe the massless spectrum of the physical theory on $M \times X$. In fact $\mathbf{D}(X)$ contains enough information to determine the tree-level superpotential of the low energy effective theory, in the form of $A_{\infty}$ products. We will discuss all of this below, but for now let us start by introducing coherent sheaves on Calabi-Yau's and del Pezzo's.

The category of coherent sheaves on a space $X$ is an enlargement of the category of vector bundles (also referred to as "locally free sheaves") on $X$ - it contains vector bundles as well as all kernels and cokernels of maps of vector bundles. For a precise definition, starting from the general concept of a sheaf, see [5] or [29]. We can very roughly think of it as including, in addition to vector bundles over $X$, more exotic objects such as vector bundles over submanifolds of $X$.

In the physical problem we consider $D$-branes on a shrinking cycle $S$ which is embedded in $X: i: S \rightarrow X$. $i$ induces an embedding $i_{*}: \mathbf{D}(S) \rightarrow \mathbf{D}(X)$, and it is no surprise that the branes we'll be interested in are in fact in the image of $i_{*}$. Now, $\mathbf{D}(S)$ has been studied extensively by mathematicians and is well understood.

We proceed by first defining a complete strongly exceptional collection of sheaves on $S$ to be an ordered set $\left\{\mathscr{F}_{0}, \cdots, \mathscr{F}_{n-1}\right\}$ that generates $\mathbf{D}(S)$ and satisfies $\operatorname{Ext}_{S}^{p}\left(\mathscr{F}_{i}, \mathscr{F}_{j}\right)=0$ for 
$p \neq 0$ and any $i$ and $j$, and $\operatorname{Ext}_{S}^{0}\left(\mathscr{F}_{i}, \mathscr{F}_{j}\right)=\operatorname{Hom}_{S}\left(\mathscr{F}_{i}, \mathscr{F}_{j}\right)=0$ for $i>j$ and $\operatorname{Hom}_{S}\left(\mathscr{F}_{i}, \mathscr{F}_{i}\right)=$ $\mathbb{C}$. Given such a complete strongly exceptional collection, we can define

$$
A=\operatorname{End}\left(\mathscr{F}_{0} \oplus \mathscr{F}_{1} \oplus \cdots \oplus \mathscr{F}_{n-1}\right)^{\text {op }}
$$

It turns out that $A$ is the path algebra of a quiver $Q$, and the $\mathscr{F}_{i}$ are isomorphic (as $A$ modules) to the projective representations $P_{i}$ defined above. Given this we can reconstruct the quiver uniquely simply by noting that $\operatorname{Hom}_{S}\left(\mathscr{F}_{i}, \mathscr{F}_{j}\right)=\operatorname{Hom}\left(P_{i}, P_{j}\right)$ is just the space of paths from node $j$ to node $i$. In fact, Bondal [26] proved that the derived category of $A$-modules, $\mathbf{D}(A$-mod $)$ is equivalent to $\mathbf{D}(S)$.

As an example, consider $S=\mathbb{P}^{2}$. An exceptional collection is given by $\{\mathscr{O}, \mathscr{O}(1), \mathscr{O}(2)\}$. We have $\operatorname{Hom}(\mathscr{O}, \mathscr{O}(1)) \cong \mathbb{C}^{3}, \operatorname{Hom}(\mathscr{O}(1), \mathscr{O}(2)) \cong \mathbb{C}^{3}$. Denote these maps, which are just multiplication by the homogeneous coordinates, by $x_{i}$ and $y_{i}$ respectively, $i=1,2,3$. We also have $\operatorname{Hom}(\mathscr{O}, \mathscr{O}(2)) \cong \mathbb{C}^{6}$ — these maps are multiplication by homogeneous degree two polynomials in the homogeneous coordinates. All this implies that we have three arrows $x_{i}$ from node 2 to node 1 , three arrows $y_{i}$ from node 3 to node 2 , and that all paths from node 3 to node 1 are compositions of these arrows, with relations $x_{i} y_{j}-x_{j} y_{i}=0$.

Another example which will be thoroughly dealt with below is $S=\mathrm{dP}_{1}$, which is $\mathbb{P}^{2}$ with one point blown up. Letting $C_{1}$ be the exceptional divisor, a complete strongly exceptional collection is $\left\{\mathscr{O}, \mathscr{O}\left(C_{1}\right), \mathscr{O}(H), \mathscr{O}(2 H)\right\}$ where $H$ is the hyperplane divisor. A slightly more involved analysis shows the quiver to be

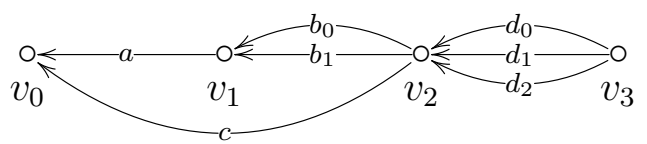

with the relations $b_{0} d_{1}-b_{1} d_{0}=0, a b_{0} d_{2}-c d_{0}=0$, and $a b_{1} d_{2}-c d_{1}=0$.

Now that we have Bondal's theorem, we can use either $\mathbf{D}(S)$ or $\mathbf{D}(A$-mod $)$ to describe branes on $S$. We will call the branes that correspond to the representations $L_{i}$ fractional branes. Of course, we are actually interested in branes in $X$, i.e., in the image of $\mathbf{D}(S)$ in $\mathbf{D}(X)$ as noted above. Using a local model of the Calabi-Yau X, namely representing it as the total space of the normal bundle of $S$ in $X$ (which is isomorphic to the canonical bundle) one can determine the Ext groups of sheaves in $i_{*} \mathbf{D}(S)$ in terms of the Ext groups in $\mathbf{D}(S)$. Namely, we find using a spectral sequence argument [21,30-32] that

$$
\operatorname{Ext}_{X}^{p}\left(i_{*} L_{i}, i_{*} L_{j}\right)=\operatorname{Ext}_{S}^{p}\left(L_{i}, L_{j}\right) \oplus \operatorname{Ext}_{S}^{3-p}\left(L_{j}, L_{i}\right)
$$

In fact it is also true that only one of the direct summands on the right hand side of the above equation is nonzero, and so we see that embedding $S$ in $X$ creates new open string degrees of freedom - new Ext ${ }^{1}$ 's corresponding to reversing Ext $^{2}$ 's in the del Pezzo quiver. We can add in arrows corresponding to these new Ext ${ }^{1}$ 's to obtain the completed quiver. For example, the completion of the Beilinson quiver is

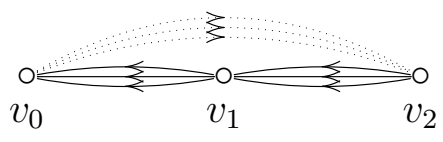


while the completion of the $\mathrm{dP}_{1}$ quiver becomes

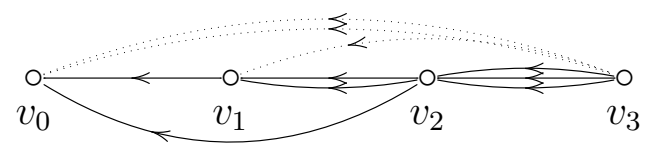

\section{Superpotentials from Topological Field Theory}

\subsection{Topological Field Theory}

Having developed and reviewed the requisite mathematical machinery, let us get to the problem at hand, namely computing superpotentials for effective dimensionally reduced theories [15]. Our setting is, as we said, $M \times X$ with $M$ being four dimensional Minkowski space and $X$ a Calabi-Yau threefold. In general, the object is to figure out how to obtain the superpotential for a specified distribution of space-filling branes - the case of interest involves putting $D 3$ branes (which look like points in $X$ ) on a collapsing del Pezzo cycle $S$ in $X$, but let us for the purpose of developing some formalism first tackle the case of a single space-filling and Calabi-Yau filling $D 9$ brane, described by a complex line bundle $E \rightarrow X$ with a hermitian connection. (By itself this case is unphysical, in a sense, because of anomalies but the topological field theory makes perfect sense.)

In this case, the massless four dimensional field content is determined by the Dolbeault cohomology of $X$ valued in $\operatorname{End}(E), H_{\bar{\partial}}^{0, q}(X, \operatorname{End}(E))$. Specifically, the number of vector bosons is given by $H_{\bar{\partial}}^{0,0}(X, \operatorname{End}(E))=\operatorname{End}(E)$, where by abuse of notation the second term refers to the space of global sections of $\operatorname{End}(E)$. We will work with simple line bundles, for which $\operatorname{End}(E)=\mathbb{C}$. We could of course also take $N$ copies of the brane, $E^{\oplus N}$, whereby we obtain a $U(N)$ gauge boson. Likewise, the number of chiral superfields is given by $H_{\bar{\partial}}^{0,1}(X, \operatorname{End}(E))$. Again, these are in the adjoint of $U(N)$ when we take the bundle to be $E^{\oplus N}$.

In order to get a term in the tree-level superpotential, we have to compute a disk diagram with boundary insertions of vertex operators that correspond to the chiral superfields that appear in that term. What makes this problem computationally tractable is the fact that this disk diagram can be computed in a topological theory [33]; it is in some sense protected from $\alpha^{\prime}$ corrections. Specifically, the open string topological $B$-model on $X$ with a $D$-brane $E$ has open string spectrum given by $A=H_{\bar{\partial}}^{0, q}(X, \operatorname{End}(E))$. Thus, if we define the disk correlation functions as:

$$
B_{i_{0}, i_{1}, \ldots, i_{k}}=(-1)^{\zeta_{1}+\zeta_{2}+\ldots+\zeta_{k-1}}\left\langle\psi_{i_{0}} \psi_{i_{1}} P \int \psi_{i_{2}}^{(1)} \int \psi_{i_{3}}^{(1)} \ldots \int \psi_{i_{k-1}}^{(1)} \psi_{i_{k}}\right\rangle,
$$

Here the $\psi_{i_{m}}$ are vertex operators of ghost number one, i.e., they correspond to states in $H_{\bar{\partial}}^{0,1}(X, \operatorname{End}(E))$. If we let $Z_{i}$ be the effective four dimensional superfield corresponding to 
the open string mode $\psi_{i}$, then the superpotential is

$$
W=\operatorname{Tr}\left(\sum_{k=2}^{\infty} \sum_{i_{0}, i_{1}, \ldots, i_{k}} \frac{B_{i_{0}, i_{1}, \ldots, i_{k}}}{k+1} Z_{i_{0}} Z_{i_{1}} \ldots Z_{i_{k}}\right) .
$$

What have we accomplished by reducing the problem to a computation in a topological sigma model? Heuristically, the situation is as follows [15]: we have, by reducing to the topological theory, essentially gotten rid of the higher mode excitations of the string. Hence the disk diagram we want is really a sum of Feynman diagrams in a field theory, called holomorphic Chern-Simons theory. Because big Feynman diagrams can be built from smaller ones, we obtain from this way of looking at things combinatorial relations among the correlators, called $A_{\infty}$ relations, and it turns out that these determine the correlators uniquely (up to field redefinition). In fact, the $A_{\infty}$ relations give a specific algorithm for generating the correlators, and this algorithm generalizes to a more general setting where $D$-branes are represented as elements of the derived category of coherent sheaves.

We now proceed to flesh out the above heuristic and describe the algorithm in detail. First, we briefly review some mathematical background on $A_{\infty}$ products.

\section{$3.2 A_{\infty}$ structure}

Given a graded vector space $B$, such as the Dolbeault complex graded by $q$ defined above, an $A_{\infty}$ structure on $B$ is defined as a series of products $m_{k}, k \geq 1$, of degree $2-k$

$$
m_{k}: B^{\otimes k} \rightarrow B
$$

which satisfy the $A_{\infty}$ constraints:

$$
\sum_{r+s+t=n}(-1)^{r+s t} m_{u}\left(\mathbf{1}^{\otimes r} \otimes m_{s} \otimes \mathbf{1}^{\otimes t}\right)=0,
$$

for any $n>0$, where $u=n+1-s$. Here we assume the usual sign rule

$$
(f \otimes g)(a \otimes b)=(-1)^{|g| \cdot|a|} f(a) \otimes g(b)
$$

when moving arguments past operators.

The $A_{\infty}$ products can actually be rephrased in terms of a differential acting on a certain space, with the complicated and unnatural looking relations between them being just the condition that the differential squares to zero [15]. We will not pursue this interpretation here however, except to note that it is useful to consider maps between spaces that commute with the differential. In terms of the $A_{\infty}$ products, such a map between two spaces $B$ and $B^{\prime}$ is described as an $A_{\infty}$ morphism, which is to say it is given by a series of maps

$$
f_{k}: B^{\otimes k} \rightarrow B^{\prime}
$$


for $k \geq 1$, which satisfy

$$
\sum_{r+s+t=n}(-1)^{r+s t} f_{u}\left(\mathbf{1}^{\otimes r} \otimes m_{s} \otimes \mathbf{1}^{\otimes t}\right)=\sum_{\substack{1 \leq r \leq n \\ i_{1}+\cdots+i_{r}=n}}(-1)^{q} m_{r}\left(f_{i_{1}} \otimes f_{i_{2}} \otimes \cdots \otimes f_{i_{r}}\right),
$$

for any $n>0, u=n+1-s$, and $q=(r-1)\left(i_{1}-1\right)+(r-2)\left(i_{2}-1\right)+\ldots+\left(i_{r-1}-1\right)$.

Now note that the $A_{\infty}$ relations give $m_{1} \cdot m_{1}=0$, so that $B$ has the structure of a graded differential complex, and we can take cohomology $H^{*}(B)$. We now come to a theorem that forms the basis for the computational tractability of our results. Let $B$ be as above, except assume that all products $m_{k}$ are zero for $k \geq 3$ - this structure is called a differential graded algebra (dga). Given an embedding $i: H^{*}(B) \rightarrow B$ Kadeishvili [34] shows that we may define an $A_{\infty}$ structure on $H^{*}(B)$ that has $m_{1}=0$ and an $A_{\infty}$ morphism $f$ from $H^{*}(B)$ to $B$ with $f_{1}$ equal to the embedding $i$. Furthermore if $B$ and $B^{\prime}$ are quasi-isomorphic dga's (that is, there is a map from one to the other that induces an isomorphism on cohomology) then the two Kadeishvili $A_{\infty}$ structures on $H^{*}(B)$ and $H^{*}\left(B^{\prime}\right)$ are $A_{\infty}$-isomorphic.

There is in fact a well defined algorithm for determining the $A_{\infty}$ products of Kadeishvili's theorem. The above condition for an $A_{\infty}$ morphism, for the case $n=2$, gives

$$
i m_{2}=(i \cdot i)+d f_{2}
$$

The cohomology class of the right hand side of the above equation is just that of $i \cdot i$ and hence $m_{2}$ is uniquely determined. Therefore $d f_{2}$ is also uniquely determined, and we can invert $d$ to obtain a (non-unique) choice of $f_{2}$. Now putting $n=3$ we have

$$
i m_{3}=f_{2}\left(\mathbf{1} \otimes m_{2}\right)-f_{2}\left(m_{2} \otimes \mathbf{1}\right)+\left(i \cdot f_{2}\right)-\left(f_{2} \cdot i\right)+d f_{3} .
$$

Once again, this equation uniquely determines $m_{3}$ and $d f_{3}$, and allows us to make a choice of $f_{3}$. Continuing in this way, it is apparent that all $A_{\infty}$ products can be determined. The ambiguity in the choice of $f_{k}$ reflects the ambiguity in the uniqueness clause of the above theorem.

\subsection{Holomorphic Chern-Simons Theory}

The field theory that the topological $B$-model on $X$ reduces to is holomorphic Chern-Simons theory:

$$
S=\int_{X} \operatorname{Tr}\left(\mathrm{A} \wedge \bar{\partial} \mathrm{A}+\frac{2}{3} \mathrm{~A} \wedge \mathrm{A} \wedge \mathrm{A}\right) \wedge \Omega,
$$

where the $\mathrm{A}$ is a $(0,1)$-form on $X$ taking values in $\operatorname{End}(E)$, and $\Omega$ is a holomorphic $(3,0)$ form on $X$. As mentioned above, computation of the disk correlator in holomorphic ChernSimons theory reduces to a sum of Feynman diagrams (this reduction can be seen explicitly as localization of the supersymmetric path integral on Feynman fat-graph configurations arising from instantons at infinity, see [35]). The combinatorial relations which the Feynman 
diagram picture gives rise to are precisely the $A_{\infty}$ relations. To make a rigorous statement, first define a trace map

$$
\gamma(a)=\int_{X} \operatorname{Tr}(a) \wedge \Omega
$$

$\gamma$ is a degree -3 map in the sense that only $(0,3)$-forms a have nonzero trace. Define $m_{1}$ to be $\bar{\partial}$ and $m_{2}$ to be the wedge product together with composition in $\operatorname{End}(E)$ - these give the Dolbeault complex the structure of a dga. The embedding of $\bar{\partial}$ cohomology into the Dolbeault complex by harmonic forms then gives via Kadeishvili an $A_{\infty}$ structure to $H_{\bar{\partial}}^{0, q}(X, \operatorname{End}(E))$. The correlation functions can then be written [36]:

$$
B_{i_{0}, i_{1}, \ldots, i_{k}}=\gamma\left(m_{2}\left(m_{k}\left(\psi_{i_{0}}, \psi_{i_{1}}, \ldots, \psi_{i_{k-1}}\right), \psi_{i_{k}}\right)\right),
$$

They satisfy the cyclicity property [36]:

$$
B_{i_{0}, i_{1}, \ldots, i_{k}}=(-1)^{\zeta_{k}\left(\zeta_{0}+\zeta_{1}+\ldots+\zeta_{k-1}\right)} B_{i_{k}, i_{0}, i_{1}, \ldots, i_{k-1}} .
$$

which will be important to us later.

Up to now we have been dealing with a single Calabi-Yau filling $D$-brane. The advantage of working in the above framework is that it extends easily to more general $D$-brane configurations. For example, (still for a single brane $E$ ) we may replace the Dolbeault complex by a Čech complex, thereby turning a difficult problem in analysis, namely inverting $\bar{\partial}$, into a more manageable combinatorial one. The uniqueness theorem above guarantees that the two $A_{\infty}$ structures obtained are $A_{\infty}$-isomorphic. We could also use an injective resolution of a sheaf instead of the Čech complex, and by appropriate abstraction reframe the entire discussion in terms of $\mathbf{D}(X)$. In fact, for now the most convenient complex for us to use is a hybrid of the Čech complex and that obtained from locally free resolutions (i.e., resolutions by vector bundles). Specifically, we claim that, for a $D$-brane represented in the derived category by the locally free resolution

$$
\mathscr{E}^{\bullet}=\left(\ldots \stackrel{\mathrm{d}_{n-2}}{\longrightarrow} \mathscr{E}^{n-1} \stackrel{\mathrm{d}_{n-1}}{\longrightarrow} \mathscr{E}^{n} \stackrel{\mathrm{d}_{n}}{\longrightarrow} \mathscr{E}^{n+1} \stackrel{\mathrm{d}_{n+1}}{\longrightarrow} \ldots\right) .
$$

the following complex has cohomology that gives the correct open string spectrum for the brane and induced $A_{\infty}$ structure that gives rise to the correct superpotential:

$$
\ldots \longrightarrow \mathscr{B}^{n-1} \longrightarrow \mathscr{B}^{n} \longrightarrow \mathscr{B}^{n+1} \longrightarrow \ldots,
$$

where

$$
\begin{aligned}
\mathscr{B}^{n} & =\bigoplus_{p+q=n} \mathscr{B}^{p, q} \\
\mathscr{B}^{p, q} & =\check{C}^{p}\left(\mathfrak{U}, \mathscr{H} o m^{q}\left(\mathscr{E}^{\bullet}, \mathscr{E}^{\bullet}\right)\right) .
\end{aligned}
$$

This is shown in [15]. Two points need to be made here. First, the differential in (32) is $d=\delta+(-1)^{p} \mathfrak{d}_{q}$, with $\delta$ the Cech differential and $\mathfrak{d}_{q}$ given by

$$
\mathfrak{d}_{n} f_{n, p}=\mathrm{d}_{p+n} \circ f_{n, p}-(-1)^{n} f_{p+1, n} \circ \mathrm{d}_{p} .
$$


where $\sum_{p} f_{n, p}$, with $f_{n, p}: \mathscr{E}^{p} \rightarrow \mathscr{E}^{p+n}$, is an element of

$$
\mathscr{H} o m^{n}(\mathscr{E} \bullet, \mathscr{E} \bullet)=\bigoplus_{p} \mathscr{H} o m\left(\mathscr{E}^{p}, \mathscr{E}^{p+n}\right)
$$

Second, to rigorously show that $\mathscr{B}$ indeed reproduces the correct spectrum and superpotential is non-trivial and requires an analysis of elements of the derived category as boundary states of the worldsheet theory [5].

\section{Superpotentials for del Pezzo singularities}

\subsection{Moduli Spaces}

Before launching into a more rigorous discussion, let us first consider a heuristic argument that will lead to a conjecture for the form of the superpotential.

First we quickly review the connection of the mathematics of quivers to the physics of D-branes and stability. One should view a quiver as representing a decay of a D-brane. The nodes in the quiver correspond to the decay products, i.e., the so-called "fractional branes" and the arrows correspond to open strings between these decay products. The D-brane we are particularly concerned with is the 3 -brane corresponding to a point in $X$.

At the instant of decay, the open strings corresponding to the arrows should be exactly massless. In the case of B-branes, these masses are a function of the complexified Kähler form $B+i J$. Here we assume that this masslessness occurs precisely when the del Pezzo surface is collapsed to a point. This assumption was justified in [37].

If one moves away from the critical point where the open strings are massless, then the D-brane may become stable or unstable with respect to the decay. If we deform the Kähler form to some generic value to give a nonzero size the del Pezzo surface (and all the curves within it) then we expect the 3-brane to be stable.

In this resolution one may compute the moduli space of the 3-brane, which should, of course, yield $X$ itself. We need not concern ourselves with the details of this process but we note the following. For more details we refer to $[3,5,37,38]$. The moduli space of 3 -branes is essentially given by the moduli space of representations of the quiver. One takes all possible quiver representations which satisfy " $\theta$-stability" and then divides by a gauge equivalence.

Physically this moduli space is given by the moduli space of chiral fields (given by the matrices associated to arrows in the quiver) corresponding to classical solutions of the field theory divided by gauge equivalence. Importantly for us, this must mean that the superpotential imposes conditions on the chiral fields equivalent to the relations in the quiver.

In other words, finding the critical points of the superpotential must be equivalent to imposing the quiver relations. This leads to an obvious proposition for the superpotential. Let $A_{i}$ be the chiral fields in the worldvolume gauge theory associated to the arrows in the (non-completed) quiver associated to a del Pezzo surface. The relations will be denoted $r_{k}\left(A_{1}, A_{2}, \ldots\right)=0$, where $r_{k}$ is some polynomial. We know from section 2.3 that each $r_{k}$ is associated to some arrow in the completed quiver, and so some chiral field $R_{k}$. It is believed 
(see $[3,5]$, for example) that in terms of the moduli space, setting all $R_{k}$ equal to zero amounts to restricting the 3-brane is be on the del Pezzo surface $S$ itself. Giving nonzero expectation values to the $R_{k}$ fields moves the 3 -brane off $S$.

If the superpotential is given by

$$
W=\sum_{k} R_{k} r_{k}\left(A_{1}, A_{2}, \ldots\right)
$$

then, on $S$, the equations of motion will yield precisely the correct constraints, at least for 3-branes on $S$. This, therefore, is our conjectured form for the superpotential.

\subsection{Quiver Gauge Theories for del Pezzos}

Let us now consider more systematically what happens when we put a D3-brane on a shrinking del Pezzo cycle $S$ in a Calabi-Yau $X$. Now, every BPS space-filling brane corresponds to a topological brane on $X$, but not vice versa. A point on $X$ is always a valid topological brane; when $S$ is of finite size, the $D 3$-brane will be located on a smooth point of $S$ and as we said we expect it to be BPS. On the other hand, when $S$ shrinks, one can argue (see e.g. [16]) that the $D 3$ is marginally stable against decay into the fractional branes introduced earlier. We think of these fractional branes as wrapping $S$ - when $S$ shrinks the point-like $D 3$ is allowed to marginally decay to them.

To get a precise description of these fractional branes, we recall that they correspond to the representations $L_{i}$, which have resolutions in terms of the projective representations $P_{i}$. If we replace the $P_{i}$ by the corresponding elements of the strongly exceptional collection (which are all vector bundles in the cases we consider) and use the equivalence between derived categories, we obtain locally free resolutions of the fractional branes. For example in the case of $\mathbb{P}^{2}$ and the Beilinson quiver, $L_{2}$ is represented in $D(S)$ as

$$
\ldots \longrightarrow 0 \longrightarrow \mathscr{F}_{0}^{\oplus 3} \stackrel{\mathrm{d}_{-3}}{\longrightarrow} \mathscr{F}_{1}^{\oplus 3} \stackrel{\mathrm{d}_{-2}}{\longrightarrow} \mathscr{F}_{2} \stackrel{\mathrm{d}-1}{\longrightarrow} 0 \longrightarrow \ldots
$$

The maps in the above complex are determined by the corresponding maps in the quiver resolution of $L_{2}$, using the fact that $\operatorname{Hom}\left(\mathscr{F}_{i}, \mathscr{F}_{j}\right)$ and $\operatorname{Hom}\left(P_{i}, P_{j}\right)$ are naturally isomorphic. It turns out that a D3-brane decays into a collection of fractional branes with each fractional brane occurring $\operatorname{dim} \mathscr{F}_{i}$ times [16]. The quiver gauge theory for a $\mathrm{dP}_{k}$ will thus have $k+3$ gauge groups, corresponding to each of the $L_{i}$, and massless matter in the bifundamental from strings stretching between $L_{i}$ and $L_{j}$. The proper setting for discussing the homological structure in this context is an $A_{\infty}$ category, but we do not need to get so abstract. We simply let $\mathscr{M}^{\bullet}$ be the direct sum of the locally free resolutions of the $L_{i}$, and use it as the starting point for the $A_{\infty}$ computations. We remind the reader that $\mathscr{M}^{\bullet}$ is a locally free resolution of sheaves over $S$, not $X$.

It will be convenient to represent $X$ as the total space of the normal bundle $N$ of $S$. Because $S$ is a del Pezzo, $N \cong K_{S}$ [16]. We are allowed to take this limit in Kähler moduli space because the tree level superpotential does not depend on Kähler parameters. 


\subsection{From Branes on $\mathrm{X}$ to Branes on $\mathrm{S}$}

The actual superpotential is computed from the $A_{\infty}$ products of the Cech complex associated to branes not on $S$, but on $X$, i.e., not from $\mathscr{B} \bullet \bullet$, but rather from the associated complex obtained from considering all the sheaves as embedded in $X$. The goal of this section is to show that the computation of $A_{\infty}$ products associated to branes on $X$ essentially reduces to the computation on $S$. Specifically, we recall that (as we will see in greater detail below) Ext $^{1}$ 's of sheaves on $S$ considered as sheaves on $X$ include all the Ext's of the sheaves on $S$ plus some extra Ext ${ }^{1}$ 's, which, after a reversal of arrows, correspond to Ext ${ }^{2}$ 's of the sheaves on $S$. We will prove that there is a choice of $A_{\infty}$ structure over $X$ such that all $A_{\infty}$ products that contain more than one of the "extra" Ext"s vanish. The products that contain no "extra" Ext"s are the same as they were over $S$, and the ones with one "extra" Ext ${ }^{1}$ are determined uniquely by the requirement of cyclicity. This, together with the fact that there are no cycles in del Pezzo quivers, will show that the superpotential is linear in the "extra" Ext $^{1} \mathrm{~s}$, with these Ext $^{1} \mathrm{~s}$ multiplying terms that are just the quiver relations.

To proceed with the proof, let $\pi: E \rightarrow S$ be the projection from the total space of $K_{S}$ to the del Pezzo $S$. We have a canonical section $\mathscr{O} \rightarrow \pi^{*} K_{S}$, given as follows: to each point in $E$ we tautologically associate a point of $S$ and an element of the fiber of $K_{S}$ over that point; this element can be viewed as an element of the fiber of $\pi^{*} K_{S}$ over the original point in $E$. Dualizing, we get a canonical map which fits into an exact sequence of sheaves

$$
0 \rightarrow \pi^{*}\left(K_{S}^{*}\right) \rightarrow \mathscr{O} \rightarrow \mathscr{O}_{S} \rightarrow 0
$$

and we can think of the first two terms as a locally free resolution of $\mathscr{O}_{S}$. The key point now is to tensor the resolution $\mathscr{M}^{\bullet}$ with the above resolution of $\mathscr{O}_{S}$ in order to obtain a locally free resolution of $i_{*} M$ :

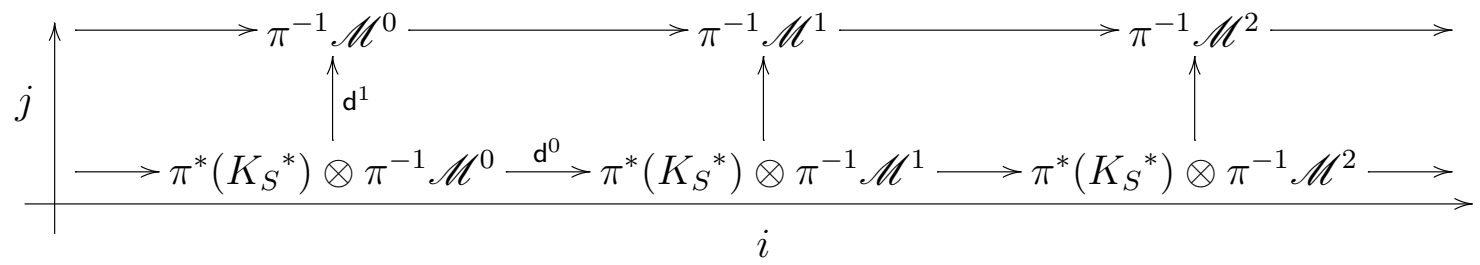

Collapsing the above double complex along the diagonal we get a free resolution, and the associated spectral sequence, which collapses at the $E_{2}$ term, shows that it is in fact a resolution of $i_{*} \mathscr{M}$. This can also be viewed as the Cone construction [5]. We will choose to retain the bi-grading, so let us represent the above resolution as $\mathscr{M}^{\bullet} \bullet$ (the first index corresponds to the index of $\mathscr{M}^{\bullet}$, and the second is either 0 , for $\pi^{-1} \mathscr{M}^{\bullet}$, or 1 , for $\pi^{*}\left(K_{S}{ }^{*}\right) \otimes$ $\left.\pi^{-1} \mathscr{M}^{\bullet}\right)$. We now define

$$
\mathscr{C}^{p, i, j}=\check{C}^{p}\left(\pi^{-1} \mathfrak{U}, \mathscr{H}_{0} m^{i, j}\left(\mathscr{M}^{\bullet, \bullet}, \mathscr{M}^{\bullet \bullet \bullet}\right)\right)
$$

Here $\mathfrak{U}$ is an affine open cover of $S$, and hence $\pi^{-1} \mathfrak{U}$ is an affine open cover of $E$. The complex $\mathscr{C}$ is central in our analysis. There are several differentials we define on $\mathscr{C}$. First of 
all, in the locally free resolution $\mathscr{M}^{\bullet \bullet \bullet}$ label the differentials that increase the first index by $\mathrm{d}_{i, j}^{0}$ and that which increases the second index by $\mathrm{d}^{1}{ }_{i, j}$. The combination $\mathrm{d}_{i, j}=\mathrm{d}_{i, j}^{0}+(-1)^{i} \mathrm{~d}_{i, j}^{1}$ is the standard differential associated to the locally free resolution of $\mathscr{M}$ over $E$. Now, given a section of $\mathscr{H}_{o m} m^{i, j}\left(\mathscr{M}^{\bullet}, \mathscr{M}^{\bullet}\right)$ over an open set $U$, i.e., a section of $\bigoplus_{p, q} \operatorname{Hom}_{U}\left(\mathscr{M}^{p, q}, \mathscr{M}^{p+i, q+j}\right)$, we can denote it by

$$
\sum_{p, q} f_{p, q}^{i, j}
$$

where

$$
f_{p, q}^{i, j}: \mathscr{M}^{p, q}(U) \rightarrow \mathscr{M}^{p+i, q+j}(U)
$$

Then we can define differentials

$$
\begin{gathered}
\mathfrak{d}^{0}{ }_{i, j} f_{p, q}^{i, j}=\mathrm{d}^{0}{ }_{i, j} f_{p, q}^{i, j}-(-1)^{(i+j)} f_{p+1, q}^{i, j} \mathrm{~d}^{0}{ }_{i, j} \\
\mathfrak{d}^{1}{ }_{i, j} f_{p, q}^{i, j}=\mathrm{d}^{1}{ }_{i, j} f_{p, q}^{i, j}-(-1)^{(i+j)} f_{p, q+1}^{i, j} \mathrm{~d}^{1}{ }_{i, j} .
\end{gathered}
$$

Finally, we have the Čech differential $\delta$, and we define the total differential on $\mathscr{C}$ by $d=\delta+(-1)^{p}\left(\mathfrak{d}^{0}+(-1)^{i} \mathfrak{d}^{1}\right)$. Now, the sum $\mathfrak{d}^{0}+(-1)^{i} \mathfrak{d}^{1}$ is the standard differential associated to the locally free resolution of $\mathscr{M}$ over $E$, so that collapsing on the $(i, j)$ indices yields the double complex in [15], showing that $\mathscr{C}$ does indeed correctly compute the $A_{\infty}$ products.

To actually get a handle on determining the $A_{\infty}$ algebra, it is useful to collapse $\mathscr{C}$ in a different way and leverage our knowledge of the $A_{\infty}$ structure for sheaves on the del Pezzo $S$. Specifically, let us collapse the complex on the $(p, i)$ indices:

$$
\mathscr{D}^{q, j}=\bigoplus_{p+i=q} \mathscr{C}^{p, i, j}
$$

$\mathscr{D}^{\bullet \bullet \bullet}$ is a double complex with anticommuting differentials $d_{0}=\delta+(-1)^{p} \mathfrak{d}^{0}$, which increases the first index, and $d_{1}=(-1)^{q} \mathfrak{d}^{1}$, which increases the second one, that add up to $d$. The desired cohomology is computed using a spectral sequence associated to this double complex, which by arguments of [16] degenerates at the $E_{2}$ term to give:

$$
\begin{array}{c|ccc}
0 & j \uparrow & 0 & 0 \\
\operatorname{Ext}_{S}^{-1}\left(M, M \otimes K_{S}\right) & \operatorname{Ext}_{S}^{0}\left(M, M \otimes K_{S}\right) & \operatorname{Ext}_{S}^{1}\left(M, M \otimes K_{S}\right) & \operatorname{Ext}_{S}^{0}\left(M, M \otimes K_{S}\right) \\
\operatorname{Ext}_{S}^{-1}(M, M) & \operatorname{Ext}_{S}^{0}(M, M) & \operatorname{Ext}_{S}^{1}(M, M) & \operatorname{Ext}_{S}^{2}(M, M) \\
\hline
\end{array}
$$

(In our exposition the $E_{1}$ term is given by taking cohomology with respect to $d_{1}$ ). Serre duality shows that $\operatorname{Ext}_{S}^{i}(M, M) \cong \operatorname{Ext}_{S}^{2-i}\left(M, M \otimes K_{S}\right)$, so that

$$
\operatorname{Ext}_{X}^{1}\left(i_{*} M, i_{*} M\right) \cong \operatorname{Ext}_{S}^{1}(M, M) \oplus \operatorname{Ext}_{S}^{2}(M, M)
$$

The two terms on the right correspond to the Ext ${ }^{1} \mathrm{~s}$ and "extra" Ext ${ }^{1} \mathrm{~s}$, respectively. 
Now, the bottom row in the above diagram reproduces the cohomology of the complex $\mathscr{B}^{p, i}$ - the complex associated to branes on $S$ rather than $X$. In fact, we may naturally embed $\mathscr{B}^{p, i}$ in

$$
\check{C}^{p}\left(\pi^{-1} \mathfrak{U}, \mathscr{H} o m^{i}\left(\pi^{-1} \mathscr{M}^{\bullet}, \pi^{-1} \mathscr{M}^{\bullet}\right)\right) .
$$

This complex, in turn, can be viewed as a sub-complex of $\mathscr{C}^{p, i, 0}$. To see why, note that, from (39), a section of $\mathscr{H} o m^{i}\left(\pi^{-1} \mathscr{M}^{\bullet}, \pi^{-1} \mathscr{M}^{\bullet}\right)$ determines a section of $\mathscr{H} o m^{i, 0}\left(\mathscr{M}^{\bullet \bullet \bullet}, \mathscr{M}^{\bullet \bullet \bullet}\right)$; basically it gives directly the maps among the sheaves in the upper row of (39), and, taking the identity map on $\pi^{*}\left(K_{S}^{*}\right) \otimes \pi^{-1} \mathscr{M}^{\bullet}$, it determines the maps for the sheaves in the lower row as well. Also, from (46), we see that the composition of these embeddings induces an isomorphism from the cohomology of $\mathscr{B}^{p, i}$ to the $j=0$ part of the cohomology of $\mathscr{C}^{p, i, j}$.

Now, in order to carry out the $A_{\infty}$ procedure we must choose representatives for all cohomology classes in $\mathscr{C}^{p, i, j}$. The upshot of the construction in the previous paragraph is that it gives us a natural choice of representatives of the $j=0$ part of the cohomology; in fact, it shows that the $A_{\infty}$ products of these $j=0$ cohomology classes are exactly the same as those in $\mathscr{B}^{p, i}$. In other words, for the $j=0$ generators the $A_{\infty}$ products are just those defined over $S$. This accomplishes part of our goal of reducing the computation over $X$ to a computation over $S$; to finish we have to deal with products that may contain some $j=1$ generators.

The $j=1$ cohomology generators are the ones that contribute the "extra" Ext ${ }^{1}$ s. To carry out the $A_{\infty}$ procedure, we must pick representatives of their cohomology classes. We choose these to be homogeneous of $j$ degree 1 , or, in other words, to lie in

$$
\check{C}^{p}\left(\pi^{-1} \mathfrak{U}, \mathscr{H} o m^{i}\left(\pi^{-1} \mathscr{M} \bullet \otimes \pi^{-1} K_{S}, \pi^{-1} \mathscr{M}^{\bullet}\right)\right)
$$

Clearly this is the most natural choice, though it should be pointed out that we could have done something stupid and chosen the generator to have a nonzero (exact) $j=0$ part, for example. The advantage of having homogeneous generators is that their products are homogeneous as well, and so vanish if they have $j>1$.

Now we claim that any $m_{k}$ that contains more than one $j=1$ generator vanishes. The naive argument would invoke the $j$ grading and the fact that there are no elements in $\mathscr{C}^{p, i, j}$ with $j>1$. The obvious flaw is the fact that $m_{k}$ does not respect the overall grading - it in fact has degree $2-k$. Thus we have to be more careful. We claim that although $m_{k}$ has nonzero degree with respect to the overall grading $p+i+j$, through a careful choice of $f_{k}$, which we now construct, we can make $m_{k}$ respect the $j$ grading. The claim at the top of this paragraph then immediately follows.

We show by induction that all the $f_{k}$ and $m_{k}$ respect the $j$ grading. Clearly $f_{1}$ and $m_{1}$ respect the $j$ grading. Suppose that this is also true for all $k \leq n$. We have for all $n+1$ (24), which can be rewritten as an equation determining $m_{n+1}$ in terms of the lower $m_{k}$ and $f_{k}$ (for $n+1=3$, for example, this is (26)). So we immediately see that it's true for $m_{n+1}$. We now deal with $f_{n+1}$. We suppress its arguments, but everywhere below $f_{n+1}$ and $d f_{n+1}$ will stand for $f_{n+1}$ and $d f_{n+1}$ applied to their arguments. Now equation (24) again gives $d f_{n+1}$ as an expression in terms of $m_{n+1}$ and the $m_{k}$ and $f_{k}$ for $k \leq n$. We have to make a choice 
of $f_{n+1}$ that respects the $j$ grading, i.e., we want $f_{n+1}$ to have the same $j$ degree as $d f_{n+1}$. Now, the case when all the arguments have $j=0$ has been discussed above and clearly we have already defined $f_{n+1}$ to have $j=0$. When more than one argument has $j=1$ then, because all the terms in the expression for $d f_{n+1}$ are homogeneous, we have $d f_{n+1}=0$, so that we can choose $f_{n+1}=0$. The nontrivial case is when exactly one argument has $j=1$. In that case, $d f_{n+1}$ is homogeneous of $j$ degree 1 and hence lies in the sub-complex

$$
\mathscr{C}^{p, i, 1}=\check{C}^{p}\left(\pi^{-1} \mathfrak{U}, \mathscr{H}_{o} m^{i}\left(\pi^{-1} \mathscr{M}^{\bullet} \otimes \pi^{-1} K_{S}, \pi^{-1} \mathscr{M}^{\bullet}\right)\right) .
$$

The crucial point is now that the embedding $\mathscr{C}^{p, i, 1} \subset \mathscr{C}^{p, i, j}$ induces an injection in cohomology. This can easily be seen from the spectral sequence (46) — the cohomology of $\mathscr{C}^{p, i, 1}$ reproduces precisely the upper, $j=1$ row in the diagram. Therefore $d f_{n+1}$ is exact not only in $\mathscr{C}^{p, i, j}$ but also in the sub-complex $\mathscr{C}^{p, i, 1}$. Therefore we can choose $f_{n+1}$ to be in $\mathscr{C}^{p, i, 1}$, so that it will have $j=1$. Thus we see that we can always choose $f_{n+1}$ to respect the $j$ grading. This completes the inductive step.

Together with the cyclicity property this determines all the $A_{\infty}$ products in $\mathscr{C}^{p, i, j}$ in terms of those over $S$. To restate, we have the original $A_{\infty}$ algebra reproduced when all the arguments are the original Ext ${ }^{1}$ 's (i.e., have $j=0$ ), any product that involves more than one "extra" Ext ${ }^{1}$ (i.e., one that has $j=1$ ) must vanish, while any product that contains

exactly one "extra" Ext ${ }^{1}$ is determined uniquely by requiring it to reproduce correlators that obey the cyclicity property (30). Having accomplished the reduction and thus shown that the superpotential is linear in the "extra" Ext ${ }^{1}$ s, we now determine the $A_{\infty}$ products over $S$ and relate them to the quiver relations.

\section{$5 \quad A_{\infty}$ relations and quivers}

We must determine the $A_{\infty}$ products over $S$, i.e., those of $\mathscr{B} \bullet \bullet$, defined in (33). We know by Bondal's theorem that $\mathbf{D}(S)$ is equivalent to $\mathbf{D}(A-\mathbf{m o d})$, where $A$ is the path algebra of the associated quiver. The operational version of this equivalence that will suffice for us is as follows. First, construct a complex of quiver representations $M^{\bullet}$ by summing the projective resolutions of the $L_{i}$. In the usual way it gives rise to the graded $\operatorname{dga} \operatorname{End}(M)^{\bullet}$. There is a natural map of this complex into $\mathscr{B}$ given by interpreting maps in $\operatorname{End}(M)$ as global sections of the $\operatorname{Hom}\left(\mathscr{F}_{i}, \mathscr{F}_{j}\right)$ and mapping them to Čech 0-cochains. Because these 0-cochains are global sections, they are annihilated by the Čech part of the differential, and one thus quickly sees that this map is a map of dga's. In fact, (the derivation of) Bondal's theorem shows that it is a quasi-isomorphism. This allows us to apply Kadeishvili's theorem and compute the $A_{\infty}$ structure of $\mathscr{B}$ in the quiver dga $\operatorname{End}(M)^{\bullet}$.

We will see that we obtain a form of the superpotential exactly as conjectured in section 4.1 


\subsection{A simple example}

We start with the Beilinson quiver, corresponding to $S=\mathbb{P}^{2}$ :

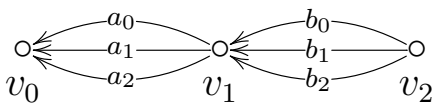

with relations $a_{\alpha} b_{\beta}=a_{\beta} b_{\alpha}$. We recall that we have the projective resolutions:

$$
\begin{array}{r}
0 \longrightarrow P_{0} \longrightarrow L_{0} \longrightarrow 0 \\
0 \longrightarrow P_{0}^{\oplus 3} \longrightarrow P_{1} \longrightarrow L_{1} \longrightarrow 0 \\
0 \longrightarrow P_{0}^{\oplus 3} \longrightarrow P_{1}^{\oplus 3} \longrightarrow P_{2} \longrightarrow L_{2} \longrightarrow 0
\end{array}
$$

and that

$$
\begin{aligned}
& \operatorname{dim} \operatorname{Ext}^{1}\left(L_{i}, L_{j}\right)=n_{i j} \\
& \operatorname{dim} \operatorname{Ext}^{2}\left(L_{i}, L_{j}\right)=r_{i j},
\end{aligned}
$$

where $n_{i j}$ counts arrows and $r_{i j}$ counts relations.

We start by choosing specific generators of the Ext ${ }^{i}$. Recalling that the Ext ${ }^{\bullet}$ can be represented as morphisms between resolutions of the $L_{i}$, we can choose the three generators $\mathrm{a}_{i}$ of $\operatorname{Ext}^{1}\left(L_{1}, L_{0}\right)$ to be

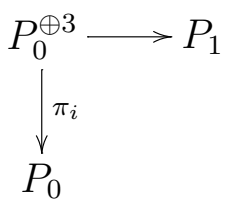

where $\pi_{i}$ is projection on the $i$ th factor. As far as the generators $\mathrm{b}_{i}$ of $\operatorname{Ext}^{1}\left(L_{2}, L_{1}\right)$ we have $\mathrm{b}_{0}$ represented as

$$
\begin{aligned}
& P_{0}^{\oplus 3} \longrightarrow P_{1}^{\oplus 3} \longrightarrow P_{2} \\
& \downarrow\left(\begin{array}{ccc}
0 & 0 & 0 \\
0 & 0 & -1 \\
0 & 1 & 0
\end{array}\right) \downarrow_{\downarrow}\left(\begin{array}{lll}
1 & 0 & 0
\end{array}\right) \\
& P_{0}^{\oplus 3} \longrightarrow P_{1}
\end{aligned}
$$

and the other $\mathbf{b}_{i}$ represented similarly. We also have the relations $\mathbf{r}_{i}$ in $\operatorname{Ext}^{2}\left(L_{3}, L_{2}\right)$, represented by

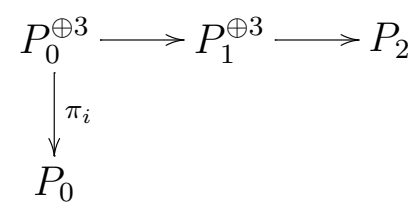

Clearly, the only potentially nontrivial products are $m_{2}\left(\mathrm{a}_{j}, \mathrm{~b}_{i}\right)$, and one easily sees by composing the representatives for $\mathrm{a}_{j}$ and $\mathrm{b}_{i}$ that $m_{2}\left(\mathrm{a}_{j}, \mathrm{~b}_{i}\right)=\epsilon^{i j k} r_{k}$. This gives rise to the superpotential

$$
W=\epsilon^{i j k} A_{i} B_{j} R_{k}
$$


which is the correct superpotential for this quiver gauge theory on the orbifold $\mathbb{C}^{3} / \mathbb{Z}_{3}[1]$. Again, the $A_{i}$ and $B_{j}$ are massless moduli corresponding to the internal structure of the shrinking cycle $\mathbb{P}^{2}$, while $R_{k}$ is the modulus that corresponds to moving the $D 3$-brane off the singularity. We note that the superpotential is of the desired form, linear in the "extra" $\operatorname{Ext}^{1} \mathrm{~s} R_{i}$, which multiply the relations. To write it out explicitly, we have

$$
W=\left(A_{0} B_{1}-B_{1} A_{0}\right) R_{2}+\left(A_{1} B_{2}-B_{2} A_{1}\right) R_{0}+\left(A_{2} B_{0}-B_{0} A_{2}\right) R_{1}
$$

\section{2 del Pezzo 1}

Let us consider the quiver associated to $\mathrm{dP}_{1}$. It is:

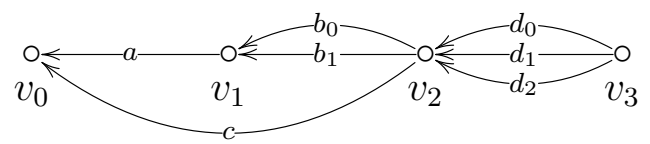

subject to the relations $r_{0}=b_{0} d_{1}-b_{1} d_{0}, s_{0}=a b_{0} d_{2}-c d_{0}=0$, and $s_{1}=a b_{1} d_{2}-c d_{1}=0$. Denote the corresponding generators of $\operatorname{Ext}^{2}$ by $r_{0}, s_{0}$, and $s_{1}$. We first pick maps of projective resolutions representing these generators, which all turn out to be uniquely determined. We have the projective resolutions:

$$
\begin{array}{r}
0 \longrightarrow P_{0} \longrightarrow L_{0} \longrightarrow 0 \\
0 \longrightarrow P_{0} \longrightarrow P_{1} \longrightarrow L_{1} \longrightarrow 0 \\
0 \longrightarrow P_{0} \oplus P_{1}^{\oplus 2} \longrightarrow P_{2} \longrightarrow L_{2} \longrightarrow 0 \\
0 \longrightarrow P_{0}^{\oplus 2} \oplus P_{1} \longrightarrow P_{2}^{\oplus 3} \longrightarrow P_{3} \longrightarrow L_{3} \longrightarrow 0
\end{array}
$$

We choose representatives of $\operatorname{Ext}^{1}\left(L_{i}, L_{j}\right)$ as follows: for $i \leq 3$, there are no relations originating at the $i$ 'th node of the quiver and hence the maps representing $\mathrm{b}_{i}$, a, and $\mathrm{c}$ are uniquely determined. The choice of representative of $d_{i}$ is uniquely determined as well. That is to say, in the diagram

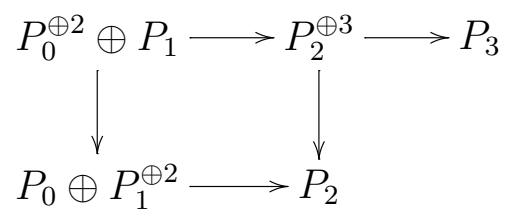

the bottom horizontal map is injective, so that the left vertical map is uniquely determined by the right vertical map, which we take to be projection on the $i$ 'th factor. Finally, for the generators of $\mathrm{Ext}^{2}$ we take the obvious uniquely determined maps from the projective resolution of $L_{3}$ to the other $L_{i}$.

We want to compute all products $m_{k}$ of the various Ext ${ }^{1}$ 's. Such products will all be in $\operatorname{Ext}^{2}$, and because only $\operatorname{Ext}^{2}\left(L_{3}, L_{0}\right)$ and $\operatorname{Ext}^{2}\left(L_{3}, L_{1}\right)$ are nonzero we see that the possible 
nonzero products are $m_{2}\left(\mathbf{b}_{i}, \mathbf{d}_{j}\right), m_{2}\left(\mathbf{c}, \mathbf{d}_{i}\right)$, and $m_{3}\left(\mathrm{a}, \mathrm{b}_{i}, \mathbf{d}_{j}\right)$. Let's look at the first of these; the relevant composition is:

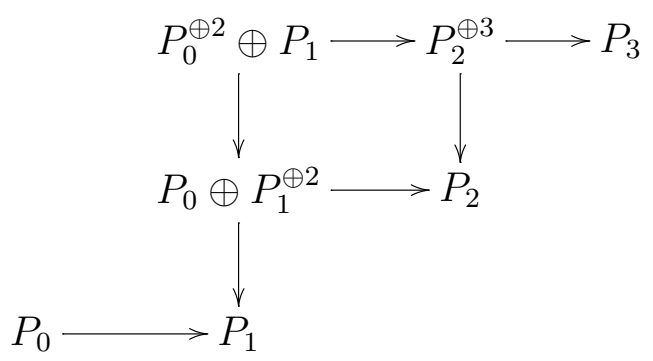

The map from the first to the second row is $\mathrm{d}_{j}$ and that from the second to the third row is $\mathrm{b}_{i}$. For convenience, we label the individual $P_{k} \mathrm{~s}$ that occur in various parts of the diagram. The upper left entry is $P_{0}^{\oplus 2} \oplus P_{1}$, where each summand corresponds to a different relation. We naturally label the two $P_{0} \mathrm{~s}$ as $S_{0}$ and $S_{1}$, and we label the $P_{1}$ as $R_{0}$. The entry below this one is $P_{0} \oplus P_{1}^{\oplus 2}$, and here each $P_{k}$ corresponds to an arrow emanating from $P_{2}$. Thus we label the $P_{0}$ as $C$ and the two $P_{1}$ s as $B_{0}$ and $B_{1}$.

Let us consider the possible maps we can have. For $k>l$ there are no nonzero maps from $P_{k}$ to $P_{l}$. From each $P_{k}$ to itself there is the identity map, and it is the only one that will be of use to us. For $k<l$, however, there are several ways to map $P_{k}$ to $P_{l}$, each corresponding to a path from node $l$ to node $k$. Thus, for example, there is one map from $P_{0}$ to $P_{1}$, denoted by $a$.

To see how $\mathrm{d}_{j}$ acts note that it maps $R_{0} \oplus S_{0} \oplus S_{1}$ to $C \oplus B_{0} \oplus B_{1}$. We can thus represent its action on $R_{0} \oplus S_{0} \oplus S_{1}$ as a 3 by 3 matrix. From the definition of $\mathrm{d}_{j}$ it is easy to obtain (by slight abuse of notation we denote by $\mathrm{d}_{j}$ both itself and its restriction to $R_{0} \oplus S_{0} \oplus S_{1}$ ):

$$
\mathrm{d}_{0}=\left(\begin{array}{ccc}
0 & -1 & 0 \\
0 & 0 & 0 \\
-1 & 0 & 0
\end{array}\right), \mathrm{d}_{1}=\left(\begin{array}{ccc}
0 & 0 & -1 \\
1 & 0 & 0 \\
0 & 0 & 0
\end{array}\right), \mathrm{d}_{2}=\left(\begin{array}{ccc}
0 & 0 & 0 \\
0 & a & 0 \\
0 & 0 & a
\end{array}\right)
$$

Note that these matrix elements are simply obtained by "contracting" the relevant relation (which indexes the column) with $d_{j}$ to obtain the elements of the column. Similar reasoning shows that the $\mathrm{b}_{i}$ act as follows:

$$
\mathrm{b}_{0}=\left(\begin{array}{lll}
0 & 1 & 0
\end{array}\right), \mathrm{b}_{1}=\left(\begin{array}{lll}
0 & 0 & 1
\end{array}\right) .
$$

The nonzero compositions are

$$
\begin{gathered}
\mathrm{b}_{0} \mathrm{~d}_{1}=\left(\begin{array}{lll}
1 & 0 & 0
\end{array}\right), \mathrm{b}_{0} \mathrm{~d}_{2}=\left(\begin{array}{lll}
0 & a & 0
\end{array}\right), \\
\mathrm{b}_{1} \mathrm{~d}_{0}=\left(\begin{array}{lll}
-1 & 0 & 0
\end{array}\right), \mathrm{b}_{1} \mathrm{~d}_{2}=\left(\begin{array}{lll}
0 & 0 & a
\end{array}\right),
\end{gathered}
$$

Now, note that the compositions $b_{0} d_{2}$ and $b_{1} d_{2}$ can both be factored through the leftmost map $P_{0} \rightarrow P_{1}$, and hence are exact in the quiver dga. So the only nonzero products are

$$
m_{2}\left(\mathrm{~b}_{0}, \mathrm{~d}_{1}\right)=\mathrm{r}_{0}, m_{2}\left(\mathrm{~b}_{1}, \mathrm{~d}_{0}\right)=-\mathrm{r}_{0}
$$


A good shorthand way of expressing this result is that $m_{2}\left(\mathrm{~b}_{i}, \mathrm{~d}_{j}\right)=\left(b_{i} d_{j}, R_{0}\right) \mathrm{r}_{0}+\left(b_{i} d_{j}, S_{0}\right) \mathrm{s}_{0}+$ $\left(b_{i} d_{j}, S_{1}\right) \mathbf{s}_{1}$, where the parentheses denote the coefficient of the string represented by the left argument in the relation represented by the right argument. As one traces through the above manipulations it is clear that this is a general result that always holds when one computes the products $m_{2}$. Thus we have $m_{2}\left(\mathrm{c}, \mathrm{d}_{0}\right)=-\mathrm{s}_{0}, m_{2}\left(\mathrm{c}, \mathrm{d}_{1}\right)=-\mathrm{s}_{1}$, and $m_{2}\left(\mathrm{c}, \mathrm{d}_{2}\right)=0$.

To compute $m_{3}$, we first have to define a choice of $f_{2}$, which must satisfy

$$
i m_{2}=(i \cdot i)+d f_{2} .
$$

Quick inspection shows that we may take $f_{2}=0$ everywhere except for $f_{2}\left(\mathrm{~b}_{i}, \mathrm{~d}_{j}\right)$. From the above analysis, we see that

$$
d f_{2}\left(\mathrm{~b}_{0}, \mathrm{~d}_{2}\right)=\left(\begin{array}{lll}
0 & -a & 0
\end{array}\right), d f_{2}\left(\mathrm{~b}_{1}, \mathrm{~d}_{2}\right)=\left(\begin{array}{lll}
0 & 0 & -a
\end{array}\right) .
$$

where $a$ denotes right multiplication and the notation indicates a map $P_{0}^{\oplus 2} \oplus P_{1} \rightarrow P_{1}$. Hence we can define $f_{2}\left(\mathrm{~b}_{0}, \mathrm{~d}_{2}\right)$ and $f_{2}\left(\mathrm{~b}_{1}, \mathrm{~d}_{2}\right)$ respectively as:

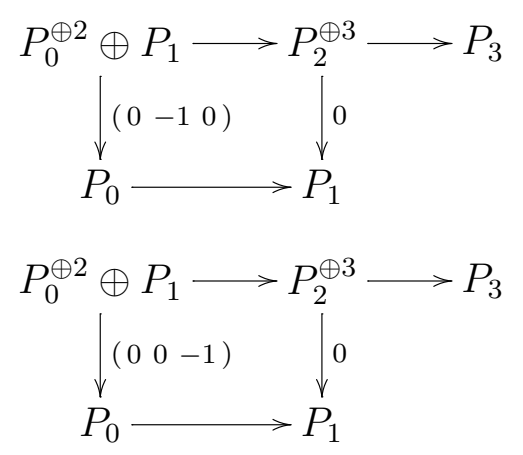

Now, we have

$$
i m_{3}=f_{2}\left(\mathbf{1} \otimes m_{2}\right)-f_{2}\left(m_{2} \otimes \mathbf{1}\right)+\left(i \cdot f_{2}\right)-\left(f_{2} \cdot i\right)+d f_{3},
$$

so that, recalling the sign rule (22),$m_{3}\left(\mathrm{a}, \mathrm{b}_{i}, \mathrm{~d}_{j}\right)=-\left[\mathrm{a} \cdot f_{2}\left(\mathrm{~b}_{i}, \mathrm{~d}_{j}\right)\right]$. Composing with a we see that $m_{3}\left(\mathrm{a}, \mathrm{b}_{0}, \mathrm{~d}_{2}\right)=\mathrm{s}_{0}$ and $m_{3}\left(\mathrm{a}, \mathrm{b}_{1}, \mathrm{~d}_{2}\right)=\mathrm{s}_{1}$. Again, a shorthand way of expressing this result is $m_{3}\left(\mathrm{a}, \mathrm{b}_{i}, \mathrm{~d}_{j}\right)=\left(a b_{i} d_{j}, R_{0}\right) \mathrm{r}_{0}+\left(a b_{i} d_{j}, S_{0}\right) \mathbf{s}_{0}+\left(a b_{i} d_{j}, S_{1}\right) \mathrm{s}_{1}$. These are all the nonzero $A_{\infty}$ products in this example.

One can see by carrying out the $A_{\infty}$ algorithm that this formula generalizes to all the $m_{k}$ in any quiver. We sketch the argument modulo various signs, which have to be checked carefully. Let the relations by labeled by $R_{i}$ and the corresponding generators of $\mathrm{Ext}^{2}$ by $\mathrm{r}_{i}$, as above. One proceeds by induction on $k_{0}$. Let's take the following inductive hypothesis: for all $j<k_{0}$, we have

$$
m_{j}\left(a_{1}, \ldots, a_{j}\right)=\sum_{i}\left(a_{1} \ldots a_{j}, R_{i}\right) r_{i}
$$

as well as a statement about $f_{k}$ for which we need to introduce some notation. Each $a_{i}$ is an $\mathrm{Ext}^{1}$, so that it can be represented as a map between the projective resolution of $L_{m(i)}$ and 
$L_{m(i-1)}$. According to this notation, $a_{i}$ is an arrow between node $m(i)$ and $m(i-1)$. The projective resolution of $L_{m(i)}$ is

$$
\cdots \longrightarrow \bigoplus_{k} P_{k}^{\oplus r_{m(i) k}} \longrightarrow \bigoplus_{k} P_{k}^{\oplus n_{m(i) k}} \longrightarrow P_{m(i)} \longrightarrow L_{m(i)} \longrightarrow 0 .
$$

The second part of the inductive hypothesis is that for $j<k_{0}, f_{j}\left(a_{1}, \ldots, a_{j}\right)$ is represented as:

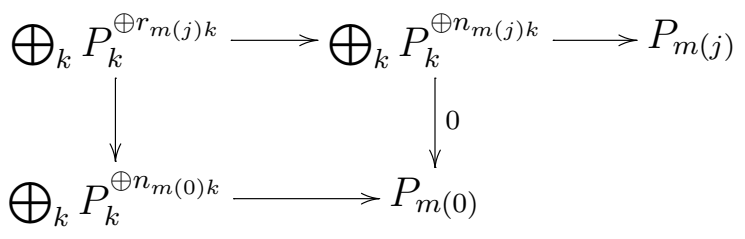

where the left vertical map takes each relation to an Ext $^{1}$ determined by the contraction of the relation with $a_{1} \ldots a_{j}$ (we extract only the linear terms in the contraction, as only these correspond to $\left.\mathrm{Ext}^{1} \mathrm{~s}\right) . f_{j}$ with any Ext ${ }^{2} \mathrm{~s}$ as arguments vanish.

For the inductive step, we have to prove the analogous statements for $m_{k_{0}}$. We use (24) to write $m_{k_{0}}$ in terms of the lower $m_{j}$ s and $f_{j}$ s. We then note from the above form of the $f_{j}$ that all terms vanish except $\left(i \cdot f_{k_{0}-1}\right)$ - basically because the only nonzero map in (75) takes relations to $\operatorname{Ext}^{1} \mathrm{~s}$, so the composition of two $f_{j}$ s vanishes. This straightforwardly leads to (73) for $m_{k_{0}}$. Inverting $d$ shows that $f_{k_{0}}$ may be chosen to be of the form (75).

Thus essentially one knows these products as soon as one knows all the relations in the quiver. It follows that the term in the superpotential that multiplies the "extra" Ext ${ }^{1}$ corresponding to a given relation is simply that relation (written as a polynomial in the Ext $\left.^{1} \mathrm{~s}\right)$. We can now write down the superpotential:

$$
W=R_{0}\left(B_{0} D_{1}-B_{1} D_{0}\right)+S_{0}\left(A B_{0} D_{2}-C D_{0}\right)+S_{1}\left(A B_{1} D_{2}-C D_{1}\right)
$$

\section{Conclusions}

We have given an effective method for computing superpotentials for quiver gauge theories associated with shrinking del Pezzo cycles. We showed that the superpotential is linear in the fields that correspond to Ext ${ }^{2} \mathrm{~s}$ in the del Pezzo quiver, and that each such field multiplies a polynomial which is just the corresponding relation. To do this we performed a precise reduction of the problem from one involving sheaves on the Calabi-Yau to one involving sheaves on the del Pezzo. We solved the problem on the del Pezzo by switching to the algebraically more tractable category of quiver representations and explicitly evaluating the $A_{\infty}$ products there. We did only the cases where $S$ is $\mathbb{P}^{2}$ and $\mathrm{dP}_{1}$, but these examples show that the algorithm is trivial to carry out provided one has the quiver and the relations. These, of course, might not be so trivial to obtain, especially for the higher del Pezzos, which themselves have complex structure moduli. These complex structure moduli are contained in the choice of points to be blown up on $\mathbb{P}^{2}$, and will show up in the quiver relations. 


\section{Acknowledgments}

We wish to thank X. Liu, J. McGreevy, A. Saltman, A. Tomasiello for useful conversations. P.S.A. is supported in part by NSF grant DMS-0301476, Stanford University, SLAC and the Packard Foundation. L.M.K. is supported by Stanford University.

\section{References}

[1] M. R. Douglas and G. Moore, D-branes, Quivers, and ALE Instantons, hep-th/9603167.

[2] D.-E. Diaconescu, M. R. Douglas, and J. Gomis, Fractional Branes and Wrapped Branes, JHEP 02 (1998) 013, hep-th/9712230.

[3] M. R. Douglas, B. Fiol, and C. Romelsberger, The Spectrum of BPS Branes on a Noncompact Calabi-Yau, hep-th/0003263.

[4] M. R. Douglas, Lectures on D-branes on Calabi-Yau manifolds, Prepared for ICTP Spring School on Superstrings and Related Matters, Trieste, Italy, 2-10 Apr 2001, http://www.ictp.trieste.it/〜 pub_off/lectures/lns007/Douglas/Douglas.pdf.

[5] P. S. Aspinwall, D-Branes on Calabi-Yau Manifolds, hep-th/0403166, to appear in the proceedings of TASI 2003.

[6] S. Franco et al., Brane Dimers and Quiver Gauge Theories, hep-th/0504110.

[7] C. V. Johnson and R. C. Myers, Aspects of Type IIB Theory on ALE Spaces, Phys. Rev. D55 (1997) 6382-6393, hep-th/9610140.

[8] I. R. Klebanov and E. Witten, Superconformal Field Theory on Threebranes at a CalabiYau Singularity, Nucl. Phys. B536 (1998) 199-218, hep-th/9807080.

[9] D. R. Morrison and M. R. Plesser, Non-Spherical Horizons. I, Adv. Theor. Math. Phys. 3 (1999) 1-81, hep-th/9810201.

[10] B. Feng, A. Hanany, and Y.-H. He, D-brane Gauge Theories from Toric Singularities and Toric Duality, Nucl. Phys. B595 (2001) 165-200, hep-th/0003085.

[11] B. Feng, A. Hanany, and Y.-H. He, Phase Structure of D-brane Gauge Theories and Toric Duality, JHEP 08 (2001) 040, hep-th/0104259.

[12] B. Feng, A. Hanany, Y.-H. He, and A. M. Uranga, Toric duality as Seiberg duality and brane diamonds, JHEP 12 (2001) 035, hep-th/0109063.

[13] B. Feng, S. Franco, A. Hanany, and Y.-H. He, Unhiggsing the del Pezzo, JHEP 08 (2003) 058, hep-th/0209228.

[14] M. Wijnholt, Large Volume Perspective on Branes at Singularities, hep-th/0212021. 
[15] P. S. Aspinwall and S. Katz, Computation of Superpotentials for D-Branes, hep-th/0412209.

[16] P. S. Aspinwall and I. V. Melnikov, D-Branes on Vanishing del Pezzo Surfaces, hep-th/0405134.

[17] C. Beasley, B. R. Greene, C. I. Lazaroiu, and M. R. Plesser, D3-branes on Partial Resolutions of Abelian Quotient Singularities of Calabi-Yau threefolds, Nucl. Phys. B566 (2000) 599-640, hep-th/9907186.

[18] C. E. Beasley and M. R. Plesser, Toric Duality is Seiberg Duality, JHEP 12 (2001) 001, hep-th/0109053.

[19] B. Feng, A. Hanany, Y. H. He, and A. Iqbal, Quiver Theories, Soliton Spectra and Picard-Lefschetz Transformations, JHEP 02 (2003) 056, hep-th/0206152.

[20] A. Hanany and A. Iqbal, Quiver Theories from D6-branes via Mirror Symmetry, JHEP 04 (2002) 009, hep-th/0108137.

[21] F. Cachazo et al., A Geometric Unification of Dualities, Nucl. Phys. B628 (2002) 3-78, hep-th/0110028.

[22] C. P. Herzog and J. Walcher, Dibaryons from Exceptional Collections, JHEP 09 (2003) 060, hep-th/0306298.

[23] C. P. Herzog, Exceptional Collections and del Pezzo Gauge Theories, hep-th/0310262.

[24] M. R. Douglas, D-Branes, Categories and N=1 Supersymmetry, J. Math. Phys. 42 (2001) 2818-2843, hep-th/0011017.

[25] P. S. Aspinwall and A. E. Lawrence, Derived Categories and Zero-Brane Stability, JHEP 08 (2001) 004, hep-th/0104147.

[26] A. I. Bondal, Representations of Associative Algebras and Coherent Sheaves, Math. USSR Izvestiya 34 (1990) 23-42.

[27] A. I. Bondal, Helices, Representations of Quivers and Koszul Algebras, in A. N. Rudakov et al., editors, "Helices and Vector Bundles", LMS Lecture Notes 148, pages 75-96, Cambridge, 1990.

[28] C. P. Herzog, Seiberg Duality is an Exceptional Mutation, hep-th/0405118.

[29] R. Hartshorne, Algebraic Geometry, Graduate Texts in Mathematics 52, SpringerVerlag, 1977.

[30] P. Seidel and R. P. Thomas, Braid Groups Actions on Derived Categories of Coherent Sheaves, Duke Math. J. 108 (2001) 37-108, math.AG/0001043. 
[31] S. Katz and E. Sharpe, D-branes, Open String Vertex Operators, and Ext Groups, Adv.Theor.Math.Phys. 6 (2003) 979-1030, hep-th/0208104.

[32] P. S. Aspinwall, A Point's Point of View of Stringy Geometry, JHEP 01 (2003) 002, hep-th/0203111.

[33] I. Brunner, M. R. Douglas, A. Lawrence, and C. Römelsberger, D-branes on the Quintic, JHEP 08 (2000) 015, hep-th/9906200.

[34] T. V. Kadeishvili, The Algebraic Structure in the Homology of an $A_{\infty}$-Algebra, Soobshch. Akad. Nauk. Gruzin. SSR 108 (1982) 249-252.

[35] E. Witten, Chern-Simons Gauge Theory as a String Theory, in H. Hofer et al., editors, "The Floer Memorial Volume", pages 637-678, Birkhäuser, 1995, hep-th/9207094.

[36] M. Herbst, C. Lazaroiu, and W. Lerche, Superpotentials, A-infinity Relations and WDVV Equations for Open Topological Strings, hep-th/0402110.

[37] P. S. Aspinwall, D-Branes, Pi-Stability and Theta-Stability, hep-th/0407123.

[38] D.-E. Diaconescu and J. Gomis, Fractional Branes and Boundary States in Orbifold Theories, JHEP 10 (2000) 001, hep-th/9906242. 